\title{
On the contribution of the $P$ and $D$ partial-wave states to the binding energy of the triton in the Bethe-Salpeter-Faddeev approach
}

\author{
S. G. Bondarenko, ${ }^{1}$ V.V. Burov ${ }^{1}$ и S. A. Yurev ${ }^{1, *}$ \\ ${ }^{1}$ Bogoliubov Laboratory of Theoretical Physics, JINR, Dubna, Russia \\ The influence of the partial-wave states with nonzero orbital moment of the nucleon \\ pair on the binding energy of the triton in the relativistic case is considered. The \\ relativistic generalization of the Faddeev equation in the Bethe-Salpeter formalism \\ is applied. Two-nucleon $t$ matrix is obtained from the Bethe-Salpeter equation with \\ separable kernel of nucleon-nucleon interaction of the rank one. The kernel form \\ factors are the relativistic type of the Yamaguchi functions. The following two-nucleon \\ partial-wave states are considered: ${ }^{1} S_{0},{ }^{3} S_{1},{ }^{3} D_{1},{ }^{3} P_{0},{ }^{1} P_{1},{ }^{3} P_{1}$. The system of the \\ integral equations are solved by using the iteration method. The binding energy of \\ the triton and three-nucleon amplitudes are found. The contribution of the $P$ and $D$ \\ states to the binding energy of triton is given.
}

\section{INTRODUCTION}

The study of three-nucleon systems has a long history and many works is devoted to the description of such nuclei. One of the most common nonrelativistic descriptions is based on the application of the Faddeev equation with various two-particle potentials. Among such potentials, there are realistic [1] and separable [2]. Such studies have made it possible to achieve significant progress in the description of static and dynamic properties of threenucleon systems.

In the same time planned experiments on the scattering of electrons by ${ }^{3} \mathrm{He}$ and ${ }^{3} \mathrm{H}$, for instance, Jefferson Lab Experiment E1210103, with the energies of the initial particles up to $12 \mathrm{GeV}$, require a relativistic description. There are ways of relativizing the non-

* yurev@jinr.ru 
relativistic description, and the methods that follow from the first principles of quantum field theory(QFT). Among the latter we single out the quasipotential Gross equation with the exchange kernel of a nucleon-nucleon interactions [3], and also a approaches based on the Bethe-Salpeter formalism with zero range of forces [4], and with a separable kernel of interaction $[5,6]$.

This work develops the ideas represented in the articles [5], where the triton is considered in the $S$-state, and [6], where along with the $S$-state, the contribution of the $D$-state into the two-particle $t$ matrix was considered. To describe a three-nucleon bound state, a relativistic generalization of Faddeev equations in the Bethe-Salpeter formalism - BetheSalpeter-Faddeev equation - is used. For simplicity of calculations, we consider nucleons have the same masses and the scalar propagators instead of the spinor ones. The spinisospin structure of the system is described through matrices of recoupling coefficient from one partial state to another.

In previous works $[7,8]$ we considered the case of taking into account the $D$-wave not only in the two-particle $t$ matrix, but also its amplitudes in the system of integral equations. In the present paper, the equation is generalized to the case of nonzero values of the angular momentum of a pair of nucleons ( $L>0: P$ - и $D$-states). The contributions of the following two-particle partial states are considered (with a full momentum of two-nucleon system $j=0,1):{ }^{1} S_{0},{ }^{3} S_{1},{ }^{3} D_{1},{ }^{3} P_{0},{ }^{1} P_{1},{ }^{3} P_{1}$. The resulting system of 12 integral equations for real and imaginary parts of amplitudes is solved by the iteration method and the binding energy of the triton, as well as all three-particle amplitudes are finding.

The work is organized as follows: after a brief description of the solution Bethe-Salpeter equations for two-nucleon states (sec. 2), the relativistic Bethe-Salpeter-Faddeev equation with scalar propagators is introdused, and the partial-wave decomposition is performed (sec. 3). In sec. 4 the results of solving the system of equations and their discussion is represent.

\section{TWO PARTICLES CASE}

Since the kernel of the Faddeev equation, written in integral form, contains a two-particle $t$ matrix we first consider the two-body problem.

The system of two relativistic particles can be described using the Bethe-Salpeter 
equation. Written for the two-particle $t$ matrix, it has the next view:

$$
\begin{aligned}
& T\left(p, p^{\prime} ; P\right)=V\left(p, p^{\prime} ; P\right)+ \\
& +\frac{i}{(2 \pi)^{4}} \int d^{4} k V(p, k ; P) G(k ; P) T\left(k, p^{\prime} ; P\right),
\end{aligned}
$$

where $p=\left(p_{1}-p_{2}\right) / 2\left[p^{\prime}=\left(p_{1}^{\prime}-p_{2}^{\prime}\right) / 2\right]$ - the relative 4-momentum of the particles of the system in the initial [final] state, $s=P^{2}$ - square of the total 4-momentum of the system $P=p_{1}+p_{2}=p_{1}^{\prime}+p_{2}^{\prime}, T\left(p, p^{\prime} ; P\right)$ - two-particle $t$ matrix, $V(p, k ; P)-$ kernel (potential) of a nucleon-nucleon $(N N)$ interaction, $G(k ; P)$ - the product of two scalar propagators of nucleons,

$$
G^{-1}(k ; P)=\left[(P / 2+k)^{2}-m_{N}^{2}+i \epsilon\right]\left[(P / 2-k)^{2}-m_{N}^{2}+i \epsilon\right] .
$$

Considering the equation (11) in the center of mass of system of two particles $P=(\sqrt{s}, \mathbf{0})$, it is possible to separate the angular dependence and carry out partial-wave decomposition:

$$
\begin{aligned}
& T_{L L^{\prime}}\left(p_{0},|\mathbf{p}|, p_{0}^{\prime},\left|\mathbf{p}^{\prime}\right| ; s\right)=V_{L L^{\prime}}\left(p_{0},|\mathbf{p}|, p_{0}^{\prime},\left|\mathbf{p}^{\prime}\right| ; s\right)+ \\
& +\frac{i}{4 \pi^{3}} \int d k_{0}|\mathbf{k}|^{2} d|\mathbf{k}| \sum_{L^{\prime \prime}} V_{L L^{\prime \prime}}\left(p_{0},|\mathbf{p}|, k_{0},|\mathbf{k}| ; s\right) G\left(k_{0},|\mathbf{k}| ; s\right) T_{L^{\prime \prime} L^{\prime}}\left(k_{0},|\mathbf{k}|, p_{0}^{\prime},\left|\mathbf{p}^{\prime}\right| ; s\right),
\end{aligned}
$$

In the present paper, for solving equation we use the kernel of the $N N$-interaction in the separable form (rank one):

$$
V_{L L^{\prime}}\left(p_{0},|\mathbf{p}|, p_{0}^{\prime},\left|\mathbf{p}^{\prime}\right| ; s\right)=\lambda g^{(L)}\left(p_{0},|\mathbf{p}|\right) g^{\left(L^{\prime}\right)}\left(p_{0}^{\prime},\left|\mathbf{p}^{\prime}\right|\right) .
$$

If we substitute in equation (3) the kernel of the $N N$-interaction as (44), then the twoparticle $t$ matrix will also have a separable form:

$$
T_{L L^{\prime}}\left(p_{0},|\mathbf{p}|, p_{0}^{\prime},\left|\mathbf{p}^{\prime}\right| ; s\right)=\tau(s) g^{(L)}\left(p_{0},|\mathbf{p}|\right) g^{\left(L^{\prime}\right)}\left(p_{0}^{\prime},\left|\mathbf{p}^{\prime}\right|\right),
$$

where function $\tau$ :

$$
\tau(s)=1 /\left(\lambda^{-1}+h(s)\right)
$$

and

$$
\begin{aligned}
& h(s)=\sum_{L} h_{L}(s)= \\
& =-\frac{i}{4 \pi^{3}} \int d k_{0} \int|\mathbf{k}|^{2} d|\mathbf{k}| \sum_{L}\left[g^{[L]}\left(k_{0},|\mathbf{k}|\right)\right]^{2} S\left(k_{0},|\mathbf{k}| ; s\right) .
\end{aligned}
$$


As form factors $g^{(L)}\left(p_{0},|\mathbf{p}|\right)$ of kernel is used relativistic generalization of the Yamaguchitype functions $[9,10]$

$$
\begin{gathered}
g^{[S]}\left(p_{0},|\mathbf{p}|\right)=\frac{1}{p_{0}^{2}-|\mathbf{p}|^{2}-\beta_{0}^{2}+i 0}, \\
g^{[P]}\left(p_{0},|\mathbf{p}|\right)=\frac{\sqrt{\left.\left|-p_{0}^{2}+\right| \mathbf{p}\right|^{2} \mid}}{\left(p_{0}^{2}-|\mathbf{p}|^{2}-\beta_{1}^{2}+i 0\right)^{2}}, \\
g^{[D]}\left(p_{0},|\mathbf{p}|\right)=\frac{C_{2}\left(p_{0}^{2}-|\mathbf{p}|^{2}\right)}{\left(p_{0}^{2}-|\mathbf{p}|^{2}-\beta_{2}^{2}+i 0\right)^{2}},
\end{gathered}
$$

where $\lambda, \beta_{0}, \beta_{1}, \beta_{2}$ и $C_{2}$ - the parameters of the model, which are selected in this way that the calculated values of the observed coincide with the corresponding experimental data for them. As observable quantities in this case can be taken the length and phase of the scattering, the effective radius, and in the case when there is a bound state - deuteron $\left({ }^{3} S_{1}-{ }^{3} D_{1}\right.$-state), - binding energy. Numerical values of parameters $\lambda$ and $\beta$ can be found in [11].

\section{THREE PARTICLES CASE}

The system of three relativistic particles can be described using the Faddeev equations in the Bethe-Salpeter formalism:

$$
\left[\begin{array}{l}
T^{(1)} \\
T^{(2)} \\
T^{(3)}
\end{array}\right]=\left[\begin{array}{c}
T_{1} \\
T_{2} \\
T_{3}
\end{array}\right]-\left[\begin{array}{ccc}
0 & T_{1} G_{1} & T_{1} G_{1} \\
T_{2} G_{2} & 0 & T_{2} G_{2} \\
T_{3} G_{3} & T_{3} G_{3} & 0
\end{array}\right]\left[\begin{array}{c}
T^{(1)} \\
T^{(2)} \\
T^{(3)}
\end{array}\right],
$$

where full $t$ matrix $T=\sum_{i=1}^{3} T^{(i)}, G_{i}$ - two-particle Green's function of particles $j$ и $n$ ((ijn) obeys cyclic permutation):

$$
G_{i}\left(k_{j}, k_{n}\right)=1 /\left(k_{j}^{2}-m_{N}^{2}+i \epsilon\right) /\left(k_{n}^{2}-m_{N}^{2}+i \epsilon\right),
$$

$T_{i}$ - two-particle $t$ matrix.

For a system of particles with the same masses, Jacobi variables can be introduced:

$$
p_{i}=\frac{1}{2}\left(k_{j}-k_{n}\right), q_{i}=\frac{1}{3} K-k_{i}, K=k_{1}+k_{2}+k_{3} .
$$

On the basis of expression (13) the equation (11) can be rewritten in the following way:

$$
\begin{aligned}
& T^{(i)}\left(p_{i}, q_{i} ; p_{i}^{\prime}, q_{i}^{\prime} ; s\right)=(2 \pi)^{4} \delta^{(4)}\left(q_{i}-q_{i}^{\prime}\right) T_{i}\left(p_{i} ; p_{i}^{\prime} ; s\right)- \\
& -i \int \frac{d p_{i}^{\prime \prime}}{(2 \pi)^{4}} T_{i}\left(p_{i} ; p_{i}^{\prime \prime} ; s\right) G_{i}\left(k_{j}^{\prime \prime}, k_{n}^{\prime \prime}\right) \times \\
& \times\left[T^{(j)}\left(p_{j}^{\prime \prime}, q_{i}^{\prime \prime} ; p_{i}^{\prime}, q_{i}^{\prime} ; s\right)+T^{(n)}\left(p_{i}^{\prime \prime}, q_{i}^{\prime \prime} ; p_{i}^{\prime}, q_{i}^{\prime} ; s\right)\right]
\end{aligned}
$$


We introduce the amplitude $\Psi^{(i)}\left(p_{i}, q_{i} ; s\right)$ for a bound three-particle state:

$$
\Psi^{(i)}\left(p_{i}, q_{i} ; s\right)=\left\langle p_{i}, q_{i}\left|T^{(i)}\right| M_{B}\right\rangle \equiv \Psi_{L M}(p, q ; s),
$$

where $M_{B}=\sqrt{s}=3 m_{N}-E_{B}$ - mass of bound state (triton), $s=K^{2}-$ square of the total momentum.

To separation the angular integration and to implement partial-wave decomposition it is need to account, that solution for the two-particle $t$ matrix is found in the system of the center of mass of two nucleons but the solution for the three-particle amplitude is sought in the center of mass system of three nucleons. Since the radial functions $g^{[L]}\left(q_{0},|\mathbf{q}|\right)$ depend on the square of the relative 4-momentum the Lorentz transformation must be carried out only for arguments of spherical harmonics. In this paper we assume, that the components of the relative 4 -vectors in the two systems coincide i.e we omit the effects of the Lorentz transformation. In this case, the dependence of the three-particle amplitudes from two 4vectors $p$ и $q$ can be divided .

We represent the total orbital angular momentum of a triton in the following form: $\boldsymbol{L}=\boldsymbol{l}+\boldsymbol{\lambda}$, where $\boldsymbol{l}$ - internal orbital angular momentum of a two-particle subsystem and $\boldsymbol{\lambda}$ - orbital angular momentum of the third particle relative to the two-particle subsystem.

In order to distinguish the explicit dependence of the amplitude on the angular momentum, we will present it in the following form:

$$
\begin{aligned}
& \Psi_{L M}(p, q ; s)=\sum_{a \lambda} \Psi_{\lambda L}^{(a)}\left(p_{0},|\mathbf{p}|, q_{0},|\mathbf{q}| ; s\right) \mathcal{Y}_{\lambda L M}^{(a)}(\hat{\mathbf{p}}, \hat{\mathbf{q}}), \\
& \mathcal{Y}_{\lambda L M}^{(a)}(\hat{\mathbf{p}}, \hat{\mathbf{q}})=\sum_{m \mu} C_{l m \lambda \mu}^{L M} Y_{l m}(\hat{\mathbf{p}}) Y_{\lambda \mu}(\hat{\mathbf{q}}),
\end{aligned}
$$

where two-nucleon states $a \equiv{ }^{2 s+1} l_{j}$ are characterized by $s$ - spin, $l$ - angular and $j$ total momentum. In the equation (16) introduced designation $\hat{\mathbf{a}} \equiv \Omega_{\mathbf{a}}$ for angular variables of 3 -vector a, $C$ - the Clebsch-Gordan coefficients, and $Y$ - spherical functions.

Using the result of the previous section for the two-particle $t$ matrix (5) and after partialwave decomposition write the amplitude $\Psi_{\lambda L}^{(a)}$ in a separable form:

$$
\begin{aligned}
& \Psi_{\lambda L}^{(a)}\left(p_{0},|\mathbf{p}|, q_{0},|\mathbf{q}| ; s\right)=g^{(a)}\left(p_{0},|\mathbf{p}|\right) \times \\
& \times \tau^{(a)}\left[\left(\frac{2}{3} \sqrt{s}+q_{0}\right)^{2}-\mathbf{q}^{2}\right] \Phi_{\lambda L}^{(a)}\left(q_{0},|\mathbf{q}| ; s\right) .
\end{aligned}
$$


The functions $\Phi_{\lambda L}^{(a)}$ satisfy the following system integral equations:

$$
\begin{aligned}
& \Phi_{\lambda L}^{(a)}\left(q_{0},|\mathbf{q}| ; s\right)=\frac{i}{4 \pi^{3}} \sum_{a^{\prime} \lambda^{\prime}} \int_{-\infty}^{\infty} d q_{0}^{\prime} \int_{0}^{\infty} \mathbf{q}^{\prime 2} d\left|\mathbf{q}^{\prime}\right| Z_{\lambda \lambda^{\prime}}^{\left(a a^{\prime}\right)}\left(q_{0}, q ; q_{0}^{\prime},\left|\mathbf{q}^{\prime}\right| ; s\right) \times \\
& \times \frac{\tau^{\left(a^{\prime}\right)}\left[\left(\frac{2}{3} \sqrt{s}+q_{0}^{\prime}\right)^{2}-\mathbf{q}^{\prime 2}\right]}{\left(\frac{1}{3} \sqrt{s}-q_{0}^{\prime}\right)^{2}-\mathbf{q}^{\prime 2}-m^{2}+i \epsilon} \Phi_{\lambda^{\prime} L}^{\left(a^{\prime}\right)}\left(q_{0}^{\prime},\left|\mathbf{q}^{\prime}\right| ; s\right),
\end{aligned}
$$

with effective kernels

$$
\begin{aligned}
& Z_{\lambda \lambda^{\prime}}^{\left(a a^{\prime}\right)}\left(q_{0},|\mathbf{q}| ; q_{0}^{\prime},\left|\mathbf{q}^{\prime}\right| ; s\right)=C_{\left(a a^{\prime}\right)} \int d \cos \vartheta_{\mathbf{q q}^{\prime}} K_{\lambda \lambda^{\prime} L}^{\left(a a^{\prime}\right)}\left(|\mathbf{q}|,\left|\mathbf{q}^{\prime}\right|, \cos \vartheta_{\mathbf{q q}^{\prime}}\right) \times \\
& \times \frac{g^{(a)}\left(-q_{0} / 2-q_{0}^{\prime},\left|\mathbf{q} / 2+\mathbf{q}^{\prime}\right|\right) g^{\left(a^{\prime}\right)}\left(q_{0}+q_{0}^{\prime} / 2,\left|\mathbf{q}+\mathbf{q}^{\prime} / 2\right|\right)}{\left(\frac{1}{3} \sqrt{s}+q_{0}+q_{0}^{\prime}\right)^{2}-\left(\mathbf{q}+\mathbf{q}^{\prime}\right)^{2}-m_{N}^{2}+i \epsilon}
\end{aligned}
$$

where

$$
\begin{aligned}
& K_{\lambda \lambda^{\prime} L}^{\left(a a^{\prime}\right)}\left(|\mathbf{q}|,\left|\mathbf{q}^{\prime}\right|, \cos \vartheta_{\mathbf{q q}^{\prime}}\right)=(4 \pi)^{3 / 2} \frac{\sqrt{2 \lambda+1}}{2 L+1} \times \\
& \times \sum_{m m^{\prime}} C_{l m \lambda 0}^{L m} C_{l^{\prime} m^{\prime} \lambda^{\prime} m-m^{\prime}}^{L m} Y_{l m}^{*}(\vartheta, 0) Y_{l^{\prime} m^{\prime}}\left(\vartheta^{\prime}, 0\right) Y_{\lambda^{\prime} m-m^{\prime}}\left(\vartheta_{\mathbf{q q}^{\prime}}, 0\right)
\end{aligned}
$$

and

$$
\begin{aligned}
& \cos \vartheta=\left(\frac{|\mathbf{q}|}{2}+\left|\mathbf{q}^{\prime}\right| \cos \vartheta_{\mathbf{q}_{\mathbf{q}^{\prime}}}\right) /\left|\frac{\mathbf{q}}{2}+\mathbf{q}^{\prime}\right| \\
& \cos \vartheta^{\prime}=\left(|\mathbf{q}|+\frac{\left|\mathbf{q}^{\prime}\right|}{2} \cos \vartheta_{\mathbf{q q}^{\prime}}\right) /\left|\mathbf{q}+\frac{\mathbf{q}^{\prime}}{2}\right|
\end{aligned}
$$

The details of the calculation of the function $K$ can be found in [12].

Since we are considering the ground state of a three-nucleon system $L=0$ and correspondingly $l=\lambda, l^{\prime}=\lambda^{\prime}$, and the function $K$ can be rewritten in the following form:

$$
\begin{aligned}
& K_{l l 0}^{\left(a a^{\prime}\right)}=\sqrt{(4 \pi)^{3}} Y_{l 0}^{*}(\vartheta, 0) A_{l}^{\prime}\left(\vartheta^{\prime}, \vartheta_{\mathbf{q q}^{\prime}}\right), \\
& A_{l}\left(\vartheta^{\prime}, \vartheta_{\mathbf{q q}^{\prime}}\right)=\sum_{m^{\prime}} C_{l m^{\prime} l-m^{\prime}}^{00} Y_{l m^{\prime}}\left(\vartheta^{\prime}, 0\right) Y_{l-m^{\prime}}\left(\vartheta_{\mathbf{q q}^{\prime}}, 0\right),
\end{aligned}
$$

where $l, l^{\prime}$ correspond to the orbital moments of the partial states $\left[a, a^{\prime}\right]$.

The accounting of spin-isospin structure of the equation kernel can be expressed in terms of matrix of recoupling coefficients from one partial state to another $\left[(a)={ }^{1}\right.$ 
$\left.S_{0},{ }^{3} S_{1},{ }^{3} D_{1},{ }^{3} P_{0},{ }^{1} P_{1},{ }^{3} P_{1}\right]$, which have the following form:

$$
C_{\left(a a^{\prime}\right)}=\frac{1}{4}\left(\begin{array}{cccccc}
1 & -3 & -3 & \sqrt{3} & -\sqrt{3} & \sqrt{3} \\
-3 & 1 & 1 & \sqrt{3} & -\sqrt{3} & \sqrt{3} \\
-3 & 1 & 1 & \sqrt{3} & -\sqrt{3} & \sqrt{3} \\
\sqrt{3} & \sqrt{3} & \sqrt{3} & -1 & -3 & -1 \\
-\sqrt{3} & -\sqrt{3} & -\sqrt{3} & -3 & -1 & -3 \\
\sqrt{3} & \sqrt{3} & \sqrt{3} & -1 & -3 & -1
\end{array}\right) .
$$

The system of integral equations (18)-(20) has singularities however, in the case of a system of three coupled particles $\left(\sqrt{s}<3 m_{N}\right)$ all these singularities do not intersect the path of integration over $q_{0}$ and thus do not affect the implementation of the procedure of Wick rotation $q_{0} \rightarrow i q_{4}$.

System (18)-(20) after Vick rotation can be solved using standard methods for solving integral equations. One of them is discussed in the next section.

\section{NUMERICAL CALCULATIONS AND RESULTS}

In this paper, a homogeneous system of 12 integral equation with a parameter, which is the binding energy of the triton, was solved using the iteration method. A homogeneous system of integral equations has a solution not for all values of parameter, but only for those that satisfy some properties.

To determine the binding energy, the following condition was used (in more detail [13]):

$$
\left.\lim _{n \rightarrow \infty} \frac{\Phi_{n}(s)}{\Phi_{n-1}(s)}\right|_{s=M_{B}^{2}}=1,
$$

where $n$-iteration number.

The procedure for solving the system of integral equations (18)-(20) by the iteration method has good convergence. In numerical calculations of the binding energy of triton and the amplitudes of its states for the Yamaguchi potential, the ratio of the previous iteration to the next did not change with the growth of the iteration number up to the sixth decimal place starting with the 20th iteration.

For the numerical calculation of the integrals, the Gauss method on a two-dimensional grid of nodes by dimension $N_{1} \times N_{2}$ was used with mapping $q_{4}=(1+x) /(1-x),|\mathbf{q}|=$ $(1+y) /(1-y)$. The influence of the number of nodes on the convergence of the result of 
numerical integration was investigated. For integration on $|\mathbf{q}|$ was enough $N_{2}=15$ nodes. With further increase quantity of nodes the numerical value of the integral did not change any more. For integration on $q_{4}$ it was not enough such quantity of nodes. For the study of convergence we increased quantity of nodes to $N_{1}=96$. With further increase quantity of nodes the numerical value of the integral changed only in the fourth decimal place. This accuracy is sufficient and allows us to take into account the contribution of various states to the binding energy.

The table represented the calculated values of the binding energy for different probabilities of the $D$-state $\left(p_{D}=4,5,6\right)$.

The values of the binding energy of a triton $(\mathrm{MeV})$

\begin{tabular}{c|c|c|c|c|c}
\hline$p_{D}$ & ${ }^{1} S_{0}-{ }^{3} S_{1}$ & ${ }^{3} D_{1}$ & ${ }^{3} P_{0}$ & ${ }^{1} P_{1}$ & ${ }^{3} P_{1}$ \\
\hline 4 & 9.221 & 9.294 & 9.314 & 9.287 & 9.271 \\
5 & 8.819 & 8.909 & 8.928 & 8.903 & 8.889 \\
6 & 8.442 & 8.545 & 8.562 & 8.540 & 8.527 \\
\hline \multicolumn{4}{c}{ Experiment } \\
\hline
\end{tabular}

The above results show that the main contribution to the binding energy of the triton give an $S$-state. Contribution of $D$-state is positive and varies from 0.8 to $1.2 \%$ depending on the probability of $D$-state in deuteron $\left(p_{D}=4-6 \%\right)$. Contributions of $P$-states have different signs and partially compensate each other, and their total contribution is $-0.2 \%$. So total contribution two-particle $P$ - and $D$-partial states with a full angular momenta $j=0,1$ into the binding energy of a triton is from 0.5 to $1 \%$. Comparison of nonrelativistic and relativistic calculations of binding energy was conducted in [5]. The paper shows that relativistic calculation of binding energy in the case of accounting only $S$-states more nonrelativistic at $0.44 \mathrm{MeV}$.

In Fig. 1 4 represented graphs of real and imaginary parts of partial amplitudes on variables $|\mathbf{q}|$ (at fixed values of $q_{4}$ ) and $q_{4}$ (at fixed values of $|\mathbf{q}|$ ). As can be seen from the graphs, amplitudes of $S$-states dominate wherein other states give a nonzero contribution. However we believe that interference contributions of $S$-, $P$ - and $D$-states in form factors of the three-particle system must be taken into account in calculations. Obtained amplitudes will be used to calculate the electromagnetic form factors of the triton using the approximations described in articles $[5,6]$. 


\section{CONCLUSION}

The solution of the relativistic Bethe - Salpeter - Faddeev equation for a three-nucleon system (triton) are considered in article. A relativistic generalization of the partial-wave decomposition procedure is carried out, which is spread to the nonzero orbital angular momenta of the interacting pair of nucleons. The case of $S$-, $P$ - and $D$-partial states of the two-particle subsystems are considered. The using of partial-wave decomposition and potential of $N N$-interactions in a separable form led to a system of integral equations for the amplitudes of states with different orbital moments of particles in the nucleus. The numerical solution of this system using the iteration method allowed find the binding energy of a triton and amplitudes of its ${ }^{1} S_{0},{ }^{3} S_{1},{ }^{3} D_{1},{ }^{3} P_{0},{ }^{1} P_{1},{ }^{3} P_{1}$-states as functions of two variables.

This work was partially supported by the Russian Foundation for Basic Research grants №16-02-00898 and №18-32-00278. 


\section{References}

1. E. van Faassen and J. A. Tjon, Phys. Rev. C 33, 2105 (1986).

2. G. Rupp, L. Streit and J. A. Tjon, Phys. Rev. C 31, 2285 (1985).

3. A. Stadler, F. Gross and M. Frank, Phys. Rev. C 56, 2396 (1997).

4. E. Ydrefors, J. H. Alvarenga Nogueira, V. Gigante, T. Frederico and V. A. Karmanov, Phys. Lett. B 770, 131 (2017).

5. G. Rupp and J. A. Tjon, Phys. Rev. C 37, 1729 (1988).

6. G. Rupp and J. A. Tjon, Phys. Rev. C 45, 2133 (1992).

7. S. G. Bondarenko, V. V. Burov and S. A. Yurev, EPJ Web Conf. 108, 02015 (2016).

8. S. Bondarenko, V. Burov and S. Yurev, EPJ Web Conf. 138, 06003 (2017).

9. Y. Yamaguchi, Phys. Rev. 95, 1628 (1954).

10. Y. Yamaguchi and Y. Yamaguchi, Phys. Rev. 95, 1635 (1954).

11. S. G. Bondarenko, V. V. Burov and S. A. Yurev, Phys.Part.Nucl.Lett. 15, 442 (2018).

12. A. Ahmadzadeh and J. A. Tjon, Phys. Rev. 139, B1085 (1965).

13. R. A. Malfliet and J. A. Tjon, Nucl. Phys. A 127, 161 (1969). 

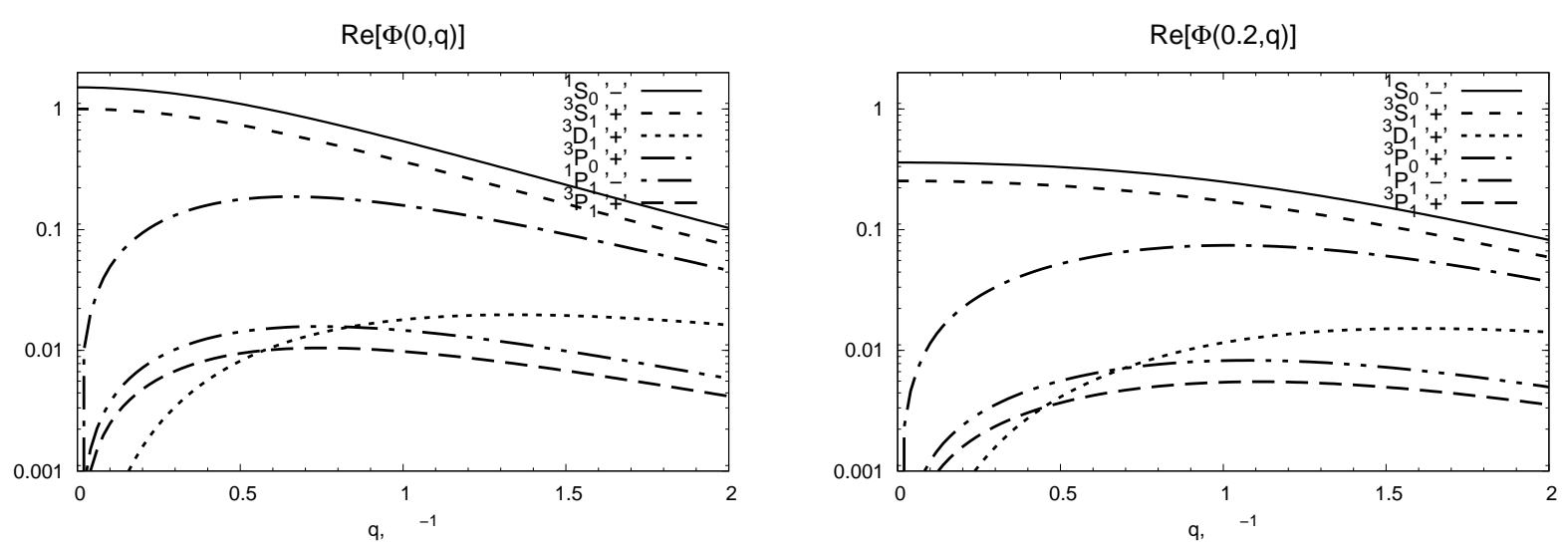

Рис. 1: The real part of the amplitudes for all states considered in the work as a function of $|\mathbf{q}|$ with the value $q_{4}=0$ and $q_{4}=0.2 \mathrm{Fm}^{-1}$.
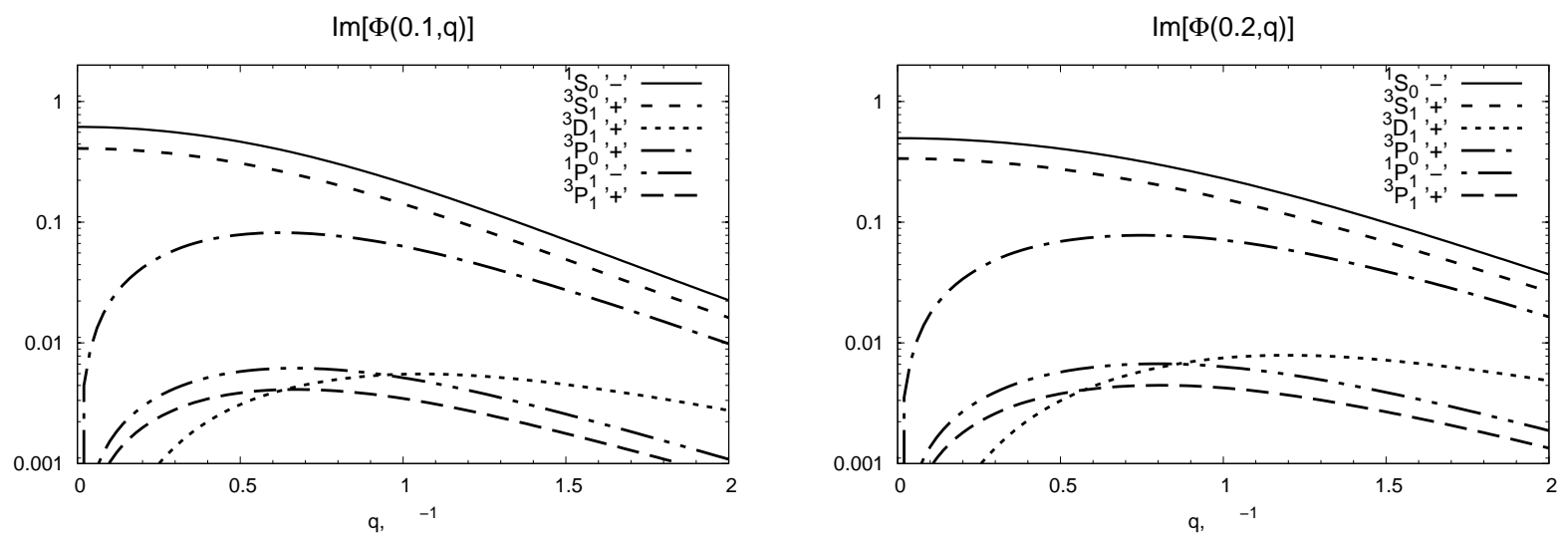

Рис. 2: The imaginary part of the amplitudes for all states considered in the work as a function of $|\mathbf{q}|$ with the value $q_{4}=0.1$ and $q_{4}=0.2 \mathrm{Fm}^{-1}$. 

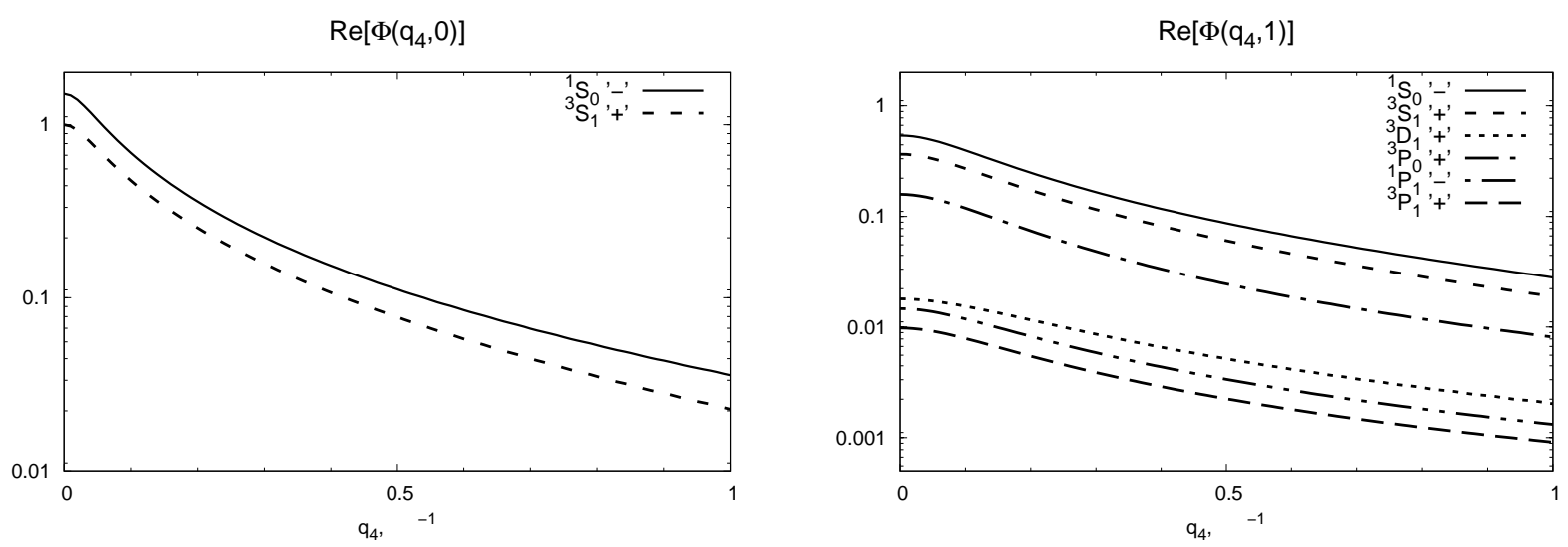

Рис. 3: The real part of the amplitudes for all states considered in the work as a function of $q_{4}$ with the value $|\mathbf{q}|=0$ and $|\mathbf{q}|=1 \mathrm{Fm}^{-1}$.
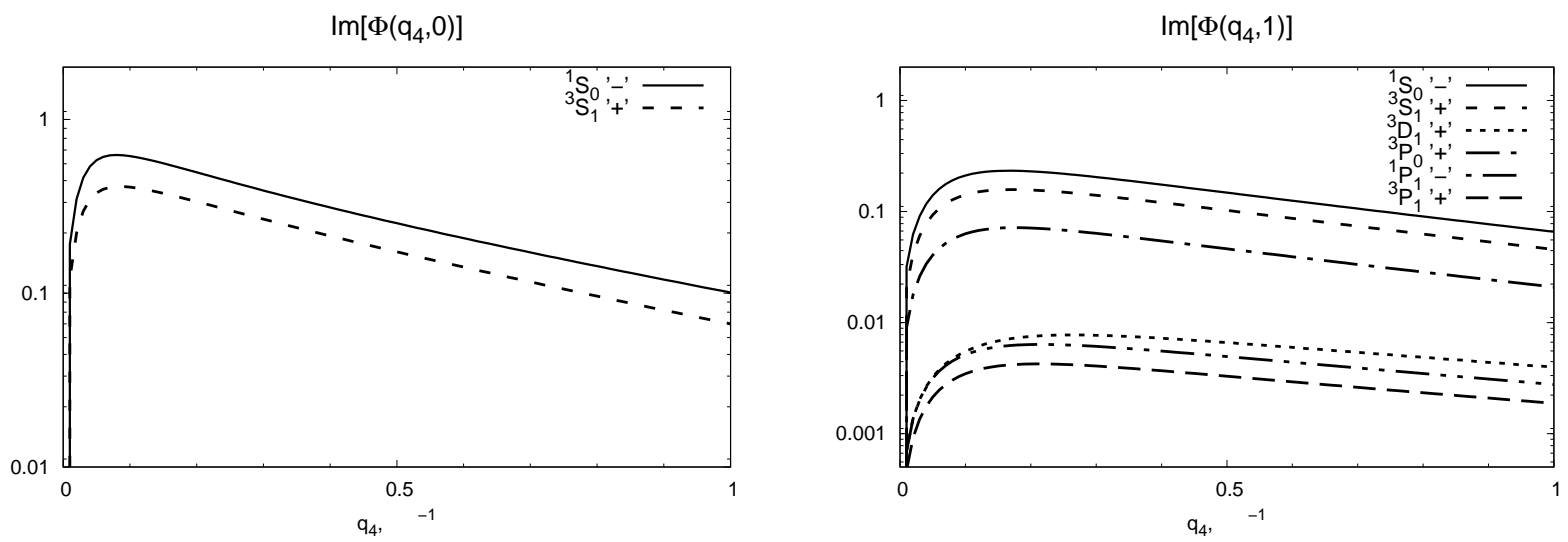

Рис. 4: The imaginary part of the amplitudes for all states considered in the work as a function of $q_{4}$ with the value $|\mathbf{q}|=0$ and $|\mathbf{q}|=1 \mathrm{Fm}^{-1}$. 